\title{
Les directives anticipées : cela ne va pas sans le dire
}

Monsieur,

J'ai relu récemment un excellent article - "À la recherche d'une bonne mort ${ }^{1} "-$, paru en juin 2005 dans le contexte d'une convergence conjoncturelle de plusieurs faits d'actualité. Il traite de la médiatisation qui a exposé au regard du public la mort d'un Pape romain - Jean-Paul II -, d'une jeune américaine - Terri Schiavo - et d'un citoyen suisse - le Fribourgeois Jean Aebischer -. Stimulante lecture pour tous les étudiants en médecine et leurs enseignants.

Tout autant que les auteurs de cet article, ainsi que des milliers de personnes dans le monde, jai été ému, intrigué, alerté, irrité par les conflits idéologiques et les luttes de pouvoir et, comme eux, j'ai perçu " la récupération de ces morts par diverses idéologies".

J'ai été inquiété par l'impact éventuel sur les citoyens "lambda" des positions souvent contradictoires des différents "pouvoirs", politique, religieux et médical, l'ensemble étant rapporté sous le contrôle incertain du "pouvoir médiatique".

J'ai eu l'impression de partager l'opinion des auteurs et cependant de ne pas arriver aux mêmes conclusions qu'eux. Je ne comprends surtout pas leur silence concernant les directives anticipées dont on ne trouve aucune mention dans leur article.

Je vais tenter d'expliquer pourquoi cette mention aurait dû découler de l'analyse des faits et suggérer que les directives anticipées méritent leur place dans le cursus des études et de la formation continue.

\section{Le "pouvoir médiatique"}

Reflétant les contradictions des autres "pouvoirs", il nous a inondés d'images montrant "le visage tordu par la douleur " du pape vivant, gardant même après la mort un "visage marqué par les stigmates de la douleur", comme le rapportait un cardinal lors d'un entretien sur une chaîne nationale de radio française, alors que, à l'inverse, gisant sur son catafalque "[...] tous les cardinaux l'on dépeint serein ", comme le rapportait la Tribune de Genève du 4 avril 2005.

Question inquiète du citoyen : les médecins ne sont-ils pas capables de contrôler la douleur?

Les photos du visage de Terri l'Américaine montraient, sans toujours préciser la date de prise de vue, soit un état souriant à la vie, soit un état très détérioré selon que le choix de la photo avait pour but de démontrer que Terri reconnaissait sa maman ou qu'il était temps de débrancher la tuyauterie.

Question inquiète du citoyen : les médecins ne savaientils pas depuis plusieurs années que ses "sourires" nétaient que des réflexes cérébraux et spinaux comme cela fut démontré par l'autopsie?

\section{Le "pouvoir politique"}

Ce "pouvoir" s'est manifesté plus vigoureusement aux Etats-Unis avec un Congrès qui a voté une loi extraordinaire permettant aux parents de Terri Schiavo d'obtenir une révision de faits (une quinzaine d'années de coma) déjà jugés par 19 juges de six cours fédérales et trois appels en cour suprême et avec la consécration finale par un aller-retour entre le Texas et Washington d'un président qui ne se dérange pas pour les morts d'enfants du Darfour. Un sénateur américain (Bill Frist, potentiel candidat à la maison blanche) n'hésite pas, le 17 mars 2005, à recommander une thérapie, sans avoir examiné Terri et sans être un spécialiste de neurologie, au simple vu d'une image présentée à la télévision du cerveau de Terri, alors que l'autopsie a indiqué que la "profonde atrophie du cerveau " indiquait "qu'aucune thérapie n'aurait permis la régénération de la perte massive de neurones".

Question inquiète du citoyen : des politiciens, élus par une majorité qui préconise une science basée sur la foi, peuvent-ils influencer mon propre "choix de ma dernière ligne droite "? 


\section{Le "pouvoir religieux"}

Il a présenté la souffrance comme une vertu. Les prêtres ont même fait des diagnostics et des recommandations thérapeutiques pour s'opposer à l'arrêt de l'alimentation artificielle. Un éthicien médical catholique et médecin du centre médical de l'Université Loyola (Chicago) condamne l'interruption de l'alimentation artificielle car "Terri ne souffre d'aucune maladie sous-jacente et cela la tuerait $"^{2}$. Cet arrêt, selon l'Osservatore Romano "condamnerait cette femme à une mort atroce, de faim et de soif".

Question inquiète du citoyen: ne dois-je pas plutôt croire le Professeur Suter, universitaire genevois spécialiste en soins intensifs, qui, selon la Tribune de Genève du 23 mars 2004, dit "il n'y a pas de souffrance au-delà de la sensation de faim et de soif que l'on ressent au début "?

\section{Le "pouvoir médical»"}

Selon ce qu'ont rapporté les médias, le "pouvoir médical» n'a pas pris de position tranchée. L'euthanasie, mot tabou, est présentée selon des définitions à géométrie variable. La diversité de situations complexes conduit à une réserve bien compréhensible concernant des actes professionnels à cheval entre l'art et la science.

Dans sa Déclaration de Venise en 1983, l'Association médicale mondiale indique "que le médecin peut épargner à un patient les souffrances d'une affection terminale, par abstentions de soins avec l'accord du patient $[\ldots]$ et lui donner les calmants propres à adoucir la phase terminale ". Malheureusement bien des textes de loi restent plus prudents et utilisent des expressions telles que : " en cas d'incertitude du pronostic " ou " $[\ldots]$ en cas de situations qui se caractérisent par une marge d'incertitude considérable " ou «[...] sous réserve qu'il ne soit pas possible de se prononcer de façon définitive " ou « [...] selon toute vraisemblance ".

Question inquiète du citoyen : respectant bien volontiers ces incertitudes, ne vous serait-il pas possible, Docteur, de m'aider mieux à exprimer mon choix dans une situation ambiguë?

La position de l'Académie suisse des sciences médicales est très claire en ce qui concerne l'utilité de "directives anticipées aussi récentes et aussi détaillées que possible $»^{45}$.

Les positions contradictoires des "pouvoirs" politiques, religieux ou médicaux ont été et sont la cause de conflits d'éthique au sein des familles et aussi an sein des équipes de soins.

Les médias nous ont montré le conflit brutal et interminable au sein de la famille de Terri, le conflit feutré et assez bien caché dans le cas de l'entourage du pape. Il semble bien que ce ne fut pas le cas avec le fribourgeois dont les derniers moments en famille furent présentés à la télévision suisse le 10 mars. La grande différence est que ce dernier avait fait, et exprimé explicitement, avec courage et lucidité, le choix de sa fin de vie.

Les auteurs de l'article font bien ressortir, en première conclusion, qu'une "bonne mort " c'est "mourir auprès des siens" en restant chez soi. Ce fut la position du Pape en refusant d'ètre hospitalisé après une première réanimation suite à un arrêt cardiaque. Pour le citoyen "lambda", rester chez soi c'est aussi augmenter les chances d'éviter l'acharnement thérapeutique car il dispose rarement d'une chambre équipée d'un bloc opératoire comme celle du pape et il est moins "vendable" pour la "presse pipeule".

\section{Pourquoi ne jamais mentionner explicitement les} deux mots "directives anticipées".

Cela me paraît incroyable et surprenant. Les auteurs sen approchent en parlant de "refuser le combat contre l'inéluctable pour faire de ses derniers instants un chemin lucide vers une mort sereine" et de "rester l'auteur du dernier chapitre de sa propre vie". Ils vont même jusqu'à mentionner "le souci d'écrire le dernier chapitre de sa vie ". Mais ils ne citent jamais les deux mots " directives anticipées". Mystère.

Ils citent les directives de l'Académie suisse des sciences médicales ${ }^{5}$ qui mentionnent "la volonté du patient " et «[...] sauf volonté contraire exprimée directement ou indirectement ". Alors pourquoi ne pas citer aussi le passage qui dit : «Dans le cas idéal, le patient a rédigé des directives anticipées aussi récentes et aussi détaillées que possible $»^{5}$ ? 
La rédaction de directives anticipées aurait permis de raccourcir la durée du calvaire de la maman de Terri. Les familles n'auraient pas eu à dépendre de la lenteur des décisions de justice car, comme le font remarquer les auteurs, "le conflit étant devenu insoluble " dans le cas "d'une personne qui ne peut plus avoir son propre avis [...] le tribunal conclut que c'est bien cela que la patiente aurait souhaité " et "décide au plus près de ce que l'on pouvait connaître de sa volonté».

\section{Cela va-t-il sans dire? Cela va mieux en le disant}

Non seulement les directives anticipées permettent à chacun d'entre nous d'exprimer nos volontés concernant les derniers moments de notre vie sur le plan biologique mais aussi sur le plan des "valeurs " comme "le choix d'être entouré par ses proches au moment de les quitter ". Ce fut le choix de Jean Aebischer, même si " certains déplorent la laïcité résolue de cette fin de vie ", en oubliant que les directives anticipées sont justement l'occasion pour un croyant de préciser l'accompagnement spirituel désiré. De même, en ce qui concerne "l'intimité qui devrait entourer de tels moments", il s'agit d'un choix personnel à préciser dans des directives. Ce ne fut pas le choix de Jean qui a estimé que la publicité servirait " comme un modèle de bien mourir".

Deux urgentistes de Lausanne concluent un article ${ }^{6}$ sur les directives anticipées par : "sans une promotion active par le monde médical, une infime minorité des patients prendront le temps d'en rédiger. Le patient a tout à gagner de ce transfert d'informations". Je me serais attendu à ce que des auteurs d'un Centre de bioéthique participent encore mieux que des urgentistes à la "promotion active" des directives anticipées. Charles-Henri Rapin et coll. ont par exemple fait de telles recommandations dans un livre récent'.

Assurément, la problématique des directives anticipées mérite d'être abordée à sa juste place dans le cursus initial des études médicales ainsi qué formation continue.

Jean-Jacques GUILBERT

5, avenue du Mail-1205 Genève Mailto:guilbertjj@yahoo.fr

\section{Références}

1. Hurst S, Mauron A. A la recherche d'une bonne mort.2005 Revue Médicale Suisse;no.22:1522-4.

2. Rosenthal E. Pope receiving food through a nasal tube. International Herald Tribune, 31 mars 2005. [On line]. Disponible sur : http://www.iht.com/articles/2005/03/30/news/pope.php

3. Association médicale mondiale. Déclaration de Venise de l'Association Médicale Mondiale sur la phase terminale de la maladie, 2003. [On-line]. Disponible sur : http://www.wma.net/flpolicy/i2.htm

4. Académie suisse des sciences médicales. Directives de l'Académie Suisse des Sciences Médicales sur la prise en charge des patientes et patients en fin de vie, 2004. [On-line]. Disponible sur : http://www.samw.ch/docs/Richtlinien/f_RL_Leben sende2004.pdf

5. Académie suisse des sciences médicales. Directives médico-éthiques de l'Académie Suisse des Sciences Médicales sur le traitement et prise en charge des patients souffrant d'atteintes cérébrales extrêmes de longue durée, 2003. [On-line]. Disponible sur: http://www.samw.ch/docs/Richtlinien/f_RL_PVS.pdf

6. Hugli O, Yersin B. Directives anticipées : un rôle clé du médecin traitant? 2004 Médecine et Hygiène ; ${ }^{\circ} 2492: 1564-7$.

7. Rapin Ch-H et coll. (sous la direction de JJ Guilbert). Stratégies pour une vieillesse réussie. Genève : Médecine et Hygiène, 2004. 